\title{
Modelling Microstructure Evolution of Ni Cermet Using a New Cellular Automaton Approach
}

\author{
Xin Wang* and Alan Atkinson \\ Department of Materials, Imperial College London SW7 2BP, UK
}

\begin{abstract}
The degradation of SOFC electrodes is often determined by their microstructure changes. It is desirable that the microstructure evolution of electrodes in service can be simulated in 3-D for real materials so that the changes in their electrochemical performance can be predicted. This involves changes in important parameters such as: electronic and ionic conductivities; TPB (triple phase boundary) density and connectivity; and tortuosity of condensed phases and porosity. In this contribution a new cellular automaton (CA) approach to simulating complex microstructure evolution is presented, which is based on free energy reduction by using an interface imbalanced interaction model. The approach is validated first in 2-D examples to reproduce wetting between two solids and grain boundary grooving, which shows the new approach is fully consistent with classical theories. The approach is then applied to simulation of the evolution of a real 3 dimensional microstructure of a Ni-YSZ cermet fuel cell anode. The modelling results show that the microstructure evolution is sensitive to the wettability of $\mathrm{Ni}$ on YSZ and that a good wettability is helpful in maintaining a slower coarsening rate and smaller reduction of electrode performance.
\end{abstract}

Keywords: Cellular Automata; Modelling; Interface Energy; Grain Growth; Morphology

\footnotetext{
*Tel: +44 20 75496809, Fax:+44 2075946757 ,

E-mail address: xin.wang@imperial.ac.uk
} 


\section{Introduction}

Solid oxide fuel cells (SOFCs) are devices for electrochemically converting chemical fuels into electricity; essentially batteries with external fuel supplies. They offer high chemical to electrical conversion efficiencies (1). SOFCs consist of four essential components: the electrolyte, the air electrode (cathode), the fuel electrode (anode), and the interconnect. They typically operate at temperatures in the range $600-950^{\circ} \mathrm{C}$, and the microstructure of SOFC materials is potentially unstable during operation due to the mobility of some of their constituent phase(s) (especially metals). Microstructure coarsening (2, 3) and interface degradation (4) have been observed in SOFCs, and lead to degradation of cell performance. The microstructure of an SOFC electrode is regarded as being just as important as its composition. This is because fuel cell electrodes often have a complex micro/nano-structure involving interconnected electronically and ionically conducting phases, gas-phase porosity, and catalytically active surfaces (5). For example, the optimization of durable efficient nickel-cermet anodes in recent decades has relied greatly on empirical improvement of materials specifications to control cermet morphology (1).

Ni-based cermets (often containing yttria-stabilised zirconia, YSZ) are the state of the art anode materials for SOFCs. The electrochemical performance of the anode is directly related to microstructure parameters such as; triple phase boundary (TPB) density, the porosity and tortuosity of solid phases and pores (6-8). Many anode microstructure models such as (9-11) are based on simplified microstructure (e.g. consisting of spherical particles). The predicted evolution of such simplified microstructure does not necessarily reflect $100 \%$ reality of any real anode which has much more complicated morphological and topological features. Coarsening of $\mathrm{Ni}$ in $\mathrm{Ni} / \mathrm{YSZ}$ anode supports has been reported using 2-D quantitative analysis of microstructure and modelled using simplified Ostwald ripening phenomenological equations [D. Simwonis, F. Tietz, and D. Stöver, "Nickel coarsening in annealed Ni/8YSZ 
anode substrates for solid oxide fuel cells," Solid State Ionics 132 (3-4), 241-251 (2000).]. However, 2-D analysis cannot address the issues of continuity in 3-D which are important for ionic conductivity in the YSZ phase, electronic conductivity in the $\mathrm{Ni}$ phase and electrochemical reaction at the TPB. Modern 3-D tomography techniques (e.g., FIB serial sectioning and x-ray tomography) allow the reconstruction and quantification of the microstructure features in great detail (6-8). In combination with real 3-D microstructure modelling, this would allow better understanding and predicting evolution of the real anode microstructure, which is essential for predicting the durability of electrodes.

Computer simulation can be a powerful tool for achieving better understanding of the complicated changes of the microstructure. A number of computer simulation methods such as: multistate kinetic Potts-Monte Carlo models (12-14); cellular automata (15-17); phase field (18-20); and topological boundary dynamics (21); have been successful in modelling recrystallization and grain growth phenomena. In the porous Ni cermet, not only grain growth, but also a number of other phenomena such as wetting/de-wetting, grooving and Ostwald ripening could occur simultaneously. The phase field method has been used in modelling the microstructure of Ni-cermet anodes by Chen et al. (19) and Jiao et al. (22). These showed how the TPB decreases with time $(19,22)$ and that contact angles have a strong effect on the microstructural evolution. In particular, reducing the contact angle of nickel on YSZ yielded less TPB reduction (19). However, Ni transport via the vapour phase was not considered in these studies $(19,22)$, although the evaporation-condensation mechanism is believed to be the major transport mechanism leading to loss of Ni surface area and reduction of the TPB density (23). Furthermore, the phase field method is computationally expensive as it requires the solution of coupled partial differential equations.

Cellular automaton (CA) methods are more efficient in modelling natural processes. This is because CA uses a numerical algorithm. It does not need to solve partial differential 
equations numerically and then transform them into difference equations in order to be suitable for numerical calculation $(15,24)$. In the CA approach, time and space are independent variables. Space is discretised into equally sized cells (voxels) and each is characterized by values defining its internal state. The system then evolves in discrete time steps according to a set of rules whose output is a function of the states of each cell and its neighbours (25-27). Thus CA simulates the evolution of complicated systems on the basis of the elementary dynamics of the interacting cells following simple generic transition rules (28). The key to the success of a CA model is in correctly transforming the underlying physical phenomena into the local transition rules.

The CA method is not only more efficient in computation resource, but also more flexible in simulating different physical phenomena, particularly when several are acting simultaneously. The CA method has been used in modeling not only recrystallization $(14,15,17,27)$ and grain growth (29-31), but also surface mass transport $(32,33)$. However, so far the transition rules used for modelling microstructure evolution appear to be rather inconsistent either in their format or in physical meaning. This has prevented its use in a system where different microstructure phenomena occur simultaneously. For example in modeling grain growth in 2D, Liu et al (29) used a deterministic CA cell transition rule based on a simple cell-counting method (i.e. counting the number of neighbouring cells having the same state as the cell being considered). Thus if a majority of cells among the cells neighbouring a given cell have the same state as the given cell, then the state of this cell will not change at the next time step. In contrast Ding et al. (31) and He et al. (30) used energy based probabilistic transition rules in modeling grain growth. Generally speaking the transition rules used in the literature fall into two categories, based on either cell counting or free energy. The cell counting approach has been used for modeling microstructure changes that are driven only by interface curvature $(15,29,32,33)$, whereas the free energy based approach has been employed in 
modelling microstructure changes driven by other driving forces, such as stored elastic energy, stored dislocation density and crystallographic orientation mismatch $(28,34,35)$. The advantage of the free energy based approach is that the transition rule can be made scalable to any mesh size and to any combination of interface mobility and energy (34). The scalability of the transition rule is very important for modelling a $\mathrm{Ni}$ cermet as different phenomena occur simultaneously. Therefore in the present study we establish cell transition rules incorporating both cell counting and free energy so that the CA method can be used reliably in modelling microstructure based on transparent underlying physical principles.

In this paper, as a modification and an improvement to the cell counting method, a mesoscale cell interaction model is introduced to relate the cell counting to the excess free energy of interface cells. This newly developed CA approach will be employed firstly to show how it replicates the simple phenomena of wetting and grooving in 2 dimensions. After that the approach will be illustrated by simulating the evolution of the experimentally determined 3-D microstructure of a typical Ni-YSZ anode. Finally, an approach to using the model for predicting the evolution of anode performance is outlined.

\section{Cell interaction model for calculating excess free energy associated with interface curvature}

According to classic continuum theories the interface energy increase due to local curvature is directly proportional to the curvature:

$$
\Delta E_{s}=\gamma \frac{c}{r} \quad \text { Eq. } 1
$$

where $C$ is a constant, $\gamma$ is the interface energy corresponding to a flat interface, and $r$ is the radius of curvature.

However, curvature is an inconvenient concept for a discrete (pixelated) space where the shape of the interface cannot be expressed analytically. A method called interpolated 
equivalent sharp surface' $(36,37)$ has previously been used to calculate the surface curvature for a discretised space, but it is difficult to extend this method from 2-D to 3-D. The cell counting method has been used to approximately calculate the surface energy $(32,33,38)$, which takes the number of air cells in the neighbourhood of a surface cell as a measure of the local curvature. However, this is a relatively crude approximation and furthermore has not been used in calculating hetero-interface or grain boundary energy which is essential for modeling multiphase materials such as Ni cermets in SOFCs. In what follows we introduce the concept of structural imbalance at an interface and from it develop a cell interaction model to calculate the interface energy arising from the local curvature and the different properties of the materials (or void) that form the interface.

The environment of interface atoms differs from that of bulk atoms in that the interface atoms have unbalanced bonding. The imbalanced atomic interactions near an interface give rise to higher energy. By analogy with this atomistic picture, a bulk CA cell (sufficiently far from any interface), with all cells in its neighbourhood occupied by the same kind of cells, has a "balanced" environment. However, compared to a bulk cell, a cell at (or near) an interface has an un-balanced arrangement of cells in its neighbourhood. In the example shown in Fig.1a, the hatched cells in the grid are the interface cells on the red phase side of the interface. When the energy of the cell ' $\mathrm{X}$ ' is to be evaluated, it is placed at the center of a virtual box, or mask, representing the range of neighbouring cells which interact with the central cell. The mask consists of a number of layers centred on the target cell as indicated by the number in Fig.1a. (If all the blue cells were replaced by red cells, the cell $\mathrm{X}$ would then become a bulk cell). In a real material, different cells would have different electronic structures (bonding), so their existence in the neighbourhood of the target cell generates an imbalance (lack of symmetry) in terms of cell interaction. Cell size can, although not 
necessarily, be much larger than an atomistic lattice unit cell. By analogy to interatomic Coulombic interaction, the "long range" interaction between any two cells can be defined as:

$$
U_{i j}=C_{o} \frac{\delta_{c_{i} c_{j}}}{r_{i j}} \quad \text { Eq. } 2
$$

where $\boldsymbol{U}_{i j}$ is the interaction potential energy, $\boldsymbol{r}_{i j}$ is the distance (all distances in the discrete CA model are measured in units of cell size, instead of actual physical length), and $C_{o}$ is an interaction parameter between two contacting cells. $\delta_{c_{i} c_{j}}$ is the Kronecker delta. When the two cells are the same ( i.e., $\boldsymbol{c}_{\boldsymbol{i}}=\boldsymbol{c}_{\boldsymbol{j}}$, where $c_{i}$ is the type of cell at position $i$ and $c_{j}$ is the type of the cell at position $j), \boldsymbol{\delta}_{\boldsymbol{c}_{i} c_{j}}=1$, otherwise $\boldsymbol{\delta}_{\boldsymbol{c}_{i} \boldsymbol{c}_{j}}=0$.

For the determination of excess energy, only the interaction between different types of cell needs to be taken into account, because cells of the same type in the neighbourhood do not contribute to the excess. In addition, by analogy with electrostatic interactions in ionic materials, we recognise that the interaction is screened by the response of other cells between the two cells being considered (e.g. dielectric polarization). To mimic this it is assumed that the contribution from the $i^{\text {th }}$ layer is inversely proportional to the total number of the cells in the volume surrounded by the $i^{\text {th }}$ layer. Therefore, the excess energy of an interface cell (e.g., cell $\mathrm{X}$ in Fig.1a) can be quantified as:

$$
U^{x}=C_{o} \sum_{i=1}^{n} \frac{\sum_{j=1}^{8 i} c_{m i s}^{i j}\left(1-\delta_{\left.c_{x} c_{i j}\right)}\right.}{V_{i} r_{i j}} \quad \text { Eq. } 3
$$

where $\boldsymbol{n}$ is the mask size (units of cell dimension, instead of physical length), $\boldsymbol{\delta}_{\boldsymbol{c}_{\boldsymbol{x}} \boldsymbol{c}_{\boldsymbol{i j}}}$ is the Kronecker delta, $\boldsymbol{C}_{\boldsymbol{m i s}}^{\boldsymbol{i} \boldsymbol{j}}$ is the "mismatch coefficient" between the cell $\boldsymbol{c}_{\boldsymbol{i} j}$ and the target cell $\boldsymbol{c}_{\boldsymbol{x}}$ (mismatch between a condensed phase cell and a void cell is taken as 1 , whereas that between different condensed phases or crystallite orientations is between 0 and 1); and $\boldsymbol{V}_{\boldsymbol{i}}$ is the "volume" enclosed by the $i^{t h}$ layer (for the $2-\mathrm{D}$ case, $\boldsymbol{V}_{i}=(\mathbf{2 i - 1})^{2}$; for the 3 -D case, $\boldsymbol{V}_{i}=(\mathbf{2 i}$ $\left.1)^{3}\right)$. The summation is carried out in layer by layer fashion and each layer has $8 \boldsymbol{i}$ cells for the 


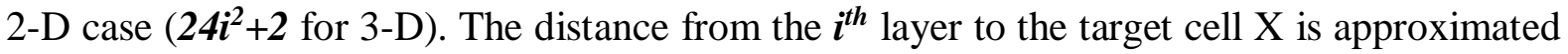
as: $\boldsymbol{r}_{i j}=\boldsymbol{i}$. This approximation and the layer-wise summation are due to a necessary correction for the artificial anisotropy, i.e., the orientation dependence of the spatial arrangement of the cells caused by the discretization. Therefore the above equation can be rewritten for 2-D:

$$
U^{x}=C_{o} \sum_{i=1}^{n} \frac{\sum_{j=1}^{8 i} c_{m i s}^{i j}\left(1-\delta_{c_{x} c_{i j}}\right)}{i(2 i-1)^{2}} \quad \text { Eq. } 4
$$

For a cell on a flat surface in 2-D (with $C_{m i s}^{i j}=1$ ) this reduces to:

$$
U^{f}=C_{o} \sum_{i=1}^{n}(4 i-1) /\left[i(2 i-1)^{2}\right] \quad \text { Eq. } 5
$$

For 3-D, the excess energy has a slightly different form:

$$
U^{x}=C_{o} \sum_{i=1}^{n} \frac{\sum_{j=1}^{24 i^{2}+2} c_{m i s}^{i j}\left(1-\delta_{\left.c_{x} c_{i j}\right)}\right.}{i\left((2 i-1)^{3}\right.} \quad \text { Eq. } 6
$$

which for a 3-D flat surface reduces to:

$$
U^{f}=C_{o} \sum_{i=1}^{n}\left[(2 i+1)^{3}-(2 i-1)^{3}-8 i\right] /\left[2 i(2 i-1)^{3}\right] \quad \text { Eq. } 7
$$

For curved surfaces, the relative structural imbalance (excess energy) with respect to the flat surface can be defined as:

$$
\Delta \boldsymbol{u}^{x}=\left(\boldsymbol{U}^{x}-\boldsymbol{U}^{f}\right) / \boldsymbol{U}^{f} \quad \text { Eq. } 8
$$

A necessary requirement for this approach is that it should reproduce the classical continuum relationship between surface energy and curvature. Thus the specific excess energies of 2-D discs of material having different radius were calculated using Eq.8 (for surface cells, the mismatch coefficient between a vacant cell and a material cell $\left.\boldsymbol{C}_{\boldsymbol{m} i \mathbf{s}}^{\boldsymbol{i} \boldsymbol{j}}=1\right)$. A disc with a radius of 70 cell units is shown Fig.1b. Fig.1c shows the calculated energies of the surface cells. It is clear from Fig.1c that surface energy is not uniform due to the discrete nature of the cell interaction model. This is in contrast to the classical continuum theory (as given by Eq.1) 
which predicts the specific surface energy for a circle would be strictly uniform on the surface. The average $\langle\boldsymbol{\Delta u}\rangle$ (taken as the sum of $\boldsymbol{\Delta u}$ over all surface cells divided by the number of surface cells) is plotted as a function of $1 / r$ in Fig.1d. It is clear that good linearity always exists between $\langle\boldsymbol{\Delta u}\rangle$ and $1 / r$, regardless of the mask size used. This fully agrees with the classical continuum theory in that specific excess energy is linearly proportional to the curvature as given in Eq.1.

In the next section, two simple 2-D microstructural evolution phenomena will be simulated as examples of the approach outlined above. The results are then compared to those of classical continuum theories which apply to these simple cases. The simulation method is then applied to a real 3-D fuel cell anode microstructure.

\section{2-D microstructure simulation}

Wetting (or de-wetting) and grooving can lead to complicated topological and morphological changes in the microstructure of a $\mathrm{Ni}$ cermet anode. Classical theories are available to describe wetting equilibrium (Young equation) and grain boundary grooving, but these deal with simple geometries and are not applicable to the complicated real geometry. In this section, wetting and grooving of simple 2-D systems will be simulated separately using the new CA approach and the results compared with the classical theories in order to validate the approach.

\section{a) Wetting}

Wetting between two solid phases depends on the compatibility of their electronic structures. In classical theory the wettability is described by the contact angle $(\boldsymbol{\theta})$, which is given by the Young equation:

$$
\gamma_{\beta G}=\gamma_{\alpha \beta}+\gamma_{\alpha G} \cos \theta
$$


where $\gamma_{\alpha G}$ and $\gamma_{\beta G}$ are the specific surface energy of the $\boldsymbol{\alpha}$ and $\boldsymbol{\beta}$ phases, respectively, and $\boldsymbol{\gamma}_{\alpha \boldsymbol{\beta}}$ is the specific interface energy between $\boldsymbol{\alpha}$ and $\boldsymbol{\beta}$ phases.

If matter transportation is via surface diffusion or evaporation/condensation, it can be simulated in the CA approach by removing matter from an occupied surface cell (which becomes vacant) and depositing it at a vacant cell elsewhere on the surface in a given time increment. Thus each time step in the CA contains two sub-steps: one for detachment of matter, and another for attachment. Here we assume that mass transportation kinetics are dominated by detachment of the molecules (cells) at the surfaces of the solids, which mimics transport by surface diffusion or evaporation/condensation when transfer is very fast and detachment/attachment is the rate controlling step. Surface cells can thus be either occupied cells or vacant cells. At each detachment step (time increment), all the occupied surface cells are allowed a chance of becoming vacant with a probability proportional to the excess energy of the cell:

$\boldsymbol{P}=\left\{\begin{array}{cc}0 & \Delta \boldsymbol{u}>0 \\ \frac{\Delta \boldsymbol{u}_{i}}{\Delta \boldsymbol{u}_{m}} & \Delta \boldsymbol{u}<0\end{array}\right.$

Where $\Delta \mathrm{u}_{\mathrm{i}} / \Delta \mathrm{u}_{\mathrm{m}}$ represents the ratio of the local and the maximum driving force (curvature, in this case).

A final decision on whether or not the filled cell will become vacant is made by a nonMetropolis Monte Carlo algorithm (35). A random number $\boldsymbol{R}(0<\boldsymbol{R}<1)$ is generated by the algorithm and if $\mathrm{R} \leq \mathrm{P}$, the state switch is accepted, but if $\mathrm{R}>\mathrm{P}$ the switch is rejected.

At each time step, after computing the detachment probabilities for the filled cells, the vacant surface cells are filled sequentially in order of decreasing energy with the detached material. If mass is conserved then the number of cells detached in the detachment step must be equal to the number of cells attached in the attachment step. If the system does not conserve mass 
then any mass loss through the vapour phase can be easily taken into account by reducing the number of cells filled in the attachment step.

Fig.2a shows an initial state of two solid particles (phase $\boldsymbol{\alpha}$ ) sitting on the top of a flat solid substrate (phase $\boldsymbol{\beta}$ ). The $\boldsymbol{\alpha}$ phase is assumed to be mobile, while the $\boldsymbol{\beta}$ phase is immobile. Figs.2b-d show the modeled morphological changes (approaching equilibrium at the three phase junction) for cases with different compatibility parameters. It is clear that the contact angle decreases as the compatibility between the phases increases as expected.

When the surface energies $\gamma_{\alpha G}$ and $\gamma_{\beta G}$ and interface energy $\gamma_{\alpha \beta}$ in the Young equation are calculated by Eq.4, 5 and 8, Eq.9 can be rewritten as:

$$
\cos \theta=2 C_{\text {com }}-1 \quad \text { Eq. } 10
$$

where $\boldsymbol{C}_{\text {com }}=1-\boldsymbol{C}_{\boldsymbol{m} i s}$. Eq.10 is plotted in Fig.2e which shows that the modelling results (data points) fit well the Young equation (solid line).

\section{b) Grain boundary grooving}

In the case of grooving (Fig.3a-e), according to the equivalent force (interface tension) point of view, the line at which a grain boundary meets a free surface is stationary at equilibrium, and therefore the forces exerted on this junction by the three interfaces must sum to zero (39). When surface energy is isotropic and independent of orientation, the geometrical dihedral angle is related to the ratio of grain boundary energy $\left(\gamma_{\mathrm{GB}}\right)$ and surface energy $\left(\gamma_{\mathrm{S}}\right)(39)$ :

$$
\frac{\gamma_{G B}}{\gamma_{S}}=2 \cos \left(\frac{\psi}{2}\right)
$$

Fig.3a shows an initial state of two adjoining grains with a grain boundary contacting a common surface at right angles. There is initially no grooving between the two grains. Figs.3b-d show the microstructure generated by the CA model using the same transition rules 
as those in modelling wetting, but with different lattice mismatch coefficients. In combination with Eqs.7, Eq. 11 can be rewritten as:

$$
\cos \left(\frac{\psi}{2}\right)=C_{m i s} \quad \text { Eq. } 12
$$

The dihedral angles obtained from the CA modelling are plotted in Fig.3e by circular datum points and Eq.12 is represented by the solid line. It is clear that the CA model results are in good agreement with the classical theory.

\section{3-D microstructure simulation}

To demonstrate how this CA model works on a simple 3-D microstructure, Fig.4a shows two nickel cubes sitting on a flat square YSZ plate. YSZ is assumed immobile at typical operating temperatures $\left(800-950^{\circ} \mathrm{C}\right)$, while $\mathrm{Ni}$ is mobile via evaporation-deposition. Fig. $4 \mathrm{~b}$ gives the CA simulated microstructure after 200 time steps assuming the mismatch coefficient between $\mathrm{Ni}$ and YSZ $\boldsymbol{C}_{\boldsymbol{m} i \mathrm{~s}}=0.8$, while Fig. 4c gives the CA simulated microstructure after 200 time steps for the case of $\boldsymbol{C}_{\boldsymbol{m} i s}=0.2$. Here $\boldsymbol{C}_{\boldsymbol{m} i \mathrm{~s}}$ represents the non-compatibility between YSZ and nickel. In the CA model, the influence of $\boldsymbol{C}_{\boldsymbol{m} \text { is }}$ on microstructure is due to its influence on the cell energy (via Eq.6). According Fig. 2e (where $\boldsymbol{C}_{\boldsymbol{c o m}}=1-\boldsymbol{C}_{\boldsymbol{m} i \boldsymbol{s}}$ ), $\boldsymbol{C}_{\boldsymbol{m} \text { is }}=0.8$ corresponds to a wetting angle of $125^{\circ}$ and 0.2 corresponds to a wetting angle of $40^{\circ}$.

From Fig.4b, it can be seen that, due to a relatively poor wettability of the Ni on YSZ, the original two cubic Ni particles have merged into one large sphere-like particle after 200 time steps. (The particle is not exactly spherical in the simulation because particle growth has reached the top of the modeled space. Due to use of a periodic boundary condition, touching at the top of the field of view means touching the bottom of the repeated YSZ plate). In contrast, the relatively good wettability (in Fig.4c) has led to a very different microstructure from Fig.4b. Therefore, the wettability of $\mathrm{Ni}$ on YSZ can be expected to play a very 
important role in determining the topological and morphological features in real anode materials.

To carry out simulation on the real microstructure of a Ni cermet anode, it is necessary to obtain the initial 3-D microstructure in digital form. For the current work, the 3-D microstructure was a sampled volume from a real anode material (as-reduced anode functional layer on an anode substrate, provided by Forschungszentrum Juelich GmbH). The 3-D microstructure reconstruction was based on sequential 2-D images obtained from slice and view experiments using dual beam focused ion beam-scanning electron microscopy (FIB-SEM, Zeiss, Auriga). Good contrast between Ni and YSZ was achieved by using an inlens detector with an acceleration voltage of 1kV. Fig.5 shows a typical 2-D image.

A series of such cross-sectional images was collected, cropped (to choose the area of interest), segmented (to distinguish different phases) and stacked to form the 3-D microstructure in digital form. Figs. 6a and $\mathrm{b}$ show the reconstructed 3-D microstructure of the anode functional layer, where the red phase is nickel and the blue phase is YSZ. The data set size is $200 \times 200 \times 100$ cells and the corresponding actual volume of this sampled section is $3 \times 3 \times$ $1.6(\mu \mathrm{m})$.

The 3-D microstructure is clearly complicated. The topology can be observed more readily from the single phase microstructure, such as Fig. $6 \mathrm{~b}$ which is a Ni-only representation after digital removal of the YSZ phase. From Fig.6b it can be seen that almost all the nickel particles are inter-linked in some way.

From the computing point of view, the cell size can vary from a few nanometers to a few microns. For practical modelling, the choice of cell size should consider the computation time (large cell size is preferable for fast modelling) and the resolution of the microstructure (small cell size is required to provide sufficient microstructure details). A general rule is that the cell 
(or voxel) size should be approximately 30 times smaller than the typical microstructure feature size (e.g. particle size or pore size). Considering that the modelled volume must be at least 8 times larger than the typical microstructure feature size in order to be representative of the global microstructure, the number of voxels for a typical evolution model can be $>13$ million. For a typical PC this took about 1-3 minutes for each modelling time step using the Matlab code in this work.

Figs. 6c and d represent the simulated microstructure after 200 time steps by assuming the mismatch coefficient $C_{m i s}=0.8$ (poor wettability), whereas Figs. 6e and f are the simulated microstructure after 200 time steps by assuming $C_{m i s}=0.2$ (good wettability). Here again a periodic boundary condition was used for the simulation. Upon comparing the evolved microstructure in Figs.6c and d $\left(C_{m i s}=0.8\right)$ with the original microstructure (Figs $6 \mathrm{a}$ and $\left.\mathrm{b}\right)$ it can be seen that some of the original ligaments between neighbouring nickel particles have been broken. Thus poor wettability of $\mathrm{Ni}$ on YSZ leads to reduced percolation of the Ni. Conversely, good compatibility $\left(C_{m i s}=0.2\right)$ encourages the spreading of the nickel phase, leading to more local linkages between neighbouring particles as shown in Figs. 6e and $6 f$.

Further important information can be obtained from quantitative analysis of the 3-D microstructures. Automated image analysis was applied to determine the volume percentage of different phases and surface and interface areas in the microstructures. For quantification of interface areas from the 3-D microstructure, interface and surface voxels need to be identified based on whether they are connected to a different phase. Voxels are considered to be connected only if they share a common face in 3-D. Linkage by an edge or a vertex is regarded as un-connected. Simply enumerating the interface voxels would overestimate the interface or surface area significantly because of the pixellation (40). In this work a 2-D layer by layer integration approach was used. In 2-D slices the interface voxels become interface 
pixels and interfaces become either lines or planes. These 2-D interface pixels are interconnected in 2-D either by sharing edges with other interface pixels (denoted type E1, E2, E3 and E4 depending on the number of shared edges) or by sharing corners (denoted type C). Hence the total interface area can be calculated by a summation:

$$
A_{\text {int }}=\sum_{k=1}^{N}\left(L(k) a_{k}+N_{E 4}(k) a_{i} a_{j}\right) \quad \text { Eq. } 13
$$

where $N$ is the total number of slices of the analysed volume, $a$ the voxel unit length and subscripts $i, j, k$ refer to three coordinate directions. $N_{\mathrm{E} 4}(k)$ is the total number of pixels of type E4 within the slice $\boldsymbol{k}$ and $L(k)=\left(1.414 N_{\mathrm{C}}+N_{\mathrm{E} 1}+N_{\mathrm{E} 2}+0.707 N_{\mathrm{E} 3}\right)\left(a_{i}+a_{j}\right) / 2$.

The results are shown in Table 1. As expected under the assumed conservation of mass, the volume percentage of different phases does not change with time, whereas the interface areas do change with time and also depend on the wettability of Ni on YSZ.

The interface area between $\mathrm{Ni}$ and pore $\left(\mathrm{SA}_{\mathrm{Ni}}\right.$, or nickel surface area) decreased nearly $18 \%$ after 200 time steps, almost independent of the wettability, whereas the interface area between YSZ and pore (SAzr, or YSZ surface area) can either increase or decrease, depending on wettability. Good wettability decreases the YSZ surface area, while poor wettability increases it. Similarly, the changes of interface area $\left(\mathrm{A}_{\mathrm{int}}\right.$ in Table 1$)$ between $\mathrm{Ni}$ and YSZ also depend on wettability: good wettability increases the contact area between the two solid phases while poor wettability decreases it.

Based on the interface areas, the equivalent particle sizes can be calculated:

$$
D_{e q}=6 V / A \quad \text { Eq. } 14
$$

where $V$ is the volume of a specific phase, $A$ is the total external area (for example, for the $\mathrm{Ni}$ phase $\left.A=S A_{N i}+A_{\text {int }}\right)$.

Table 2 lists the equivalent particle size for the three different phases. Since YSZ is set as immobile in the CA model, its particle size is unchanged (as expected). However, poor 
wettability leads to a coarsening of the $\mathrm{Ni}$ phase and a smaller average pore size, whereas good wettability leads to a smaller Ni particle size and larger pore size.

TPB length density is directly related to the electrochemical performance of the Ni-YSZ cermet anode as explained later. For TPB to be electrochemically active, the transporting species (electrons, ions and fuel) must be able to diffuse/conduct from the appropriate side of the electrode to the TPB. Otherwise, the TPB is isolated and it does not contribute to the electrode performance (11). Therefore TPBs are considered to be percolating only if there is a continuous network of ionic, electronic and pore phases extending across the electrode bulk (from the appropriate external source or sink of reacting species) to the TPB point of interest (41). In this work, TBP length was calculated by first identifying the solid hetero-interfaces by inserting an interstitial layer between any two contacting material layers. Then the TPB cells in these interstitial layers (which neighbour three different types of cells: YSZ, Ni and void) were located, the percolating TPB cells identified and the length of percolating TPB calculated. Simply counting TPB cells (whether percolating or in total) will overestimate TPB length because of the inevitable straight edges that arise from the discretization of the cells. All turning corners were adjusted for discretisation. For example if the turning is in the i-j plane, the length will be $\left(a_{i}+a_{j}\right) / 2+\left(a_{i}^{2}+a_{j}^{2}\right)^{0.5} / 2$ instead of $a_{i}+a_{j}$ ( $a$ being voxel length, and $i$ and $j$ are any two different coordinate directions ). To determine the percolating TPB densities, the connectivity of each individual phase contacting each TPB segment was checked. Any YSZ voxel not connected through the YSZ to the electrolyte side of the analysed volume was regarded as inert and all TPB cells contacting the "inert phase" were considered as non-percolating. Similar criteria were used for the Ni and pore phases, but considering their connectivity with the opposite face of the analysed volume, which is the external source for the electrons and fuel. In other words only those TPB segments which are associated with percolating voxels were counted as percolating. Due to the small sampled 
volume, the percolating TPB length determined in this way is likely to be somewhat smaller than that in reality. This is because some of the voxels, especially those near the edge (vertical) boundaries of the sampled volume may be connected via the space outside the sampled volume. With an increased sampled volume, this uncertainty will be decreased, but this will increase computation cost. The optimum sample volume is currently not known, but the present results serve to illustrate the changes in TPB that are likely.

The percolating and total TPB densities for the different evolved microstructures are given in Table 3. The poor wettability $\left(C_{m i s}=0.8\right)$ has led to decreases of both percolating TPB density (from 5.16 to $4.06 \mu \mathrm{m} / \mu \mathrm{m}^{3}$ ) and total TPB density (from 6.14 to $5.09 \mu \mathrm{m} / \mu \mathrm{m}^{3}$ ). In contrast, a good wettability $\left(C_{m i s}=0.2\right)$ has led to a big increase of the total TPB density (6.14 to $10.6 \mu \mathrm{m} / \mu \mathrm{m}^{3}$ ) but a decrease of the percolating TPB density (from 5.16 to 4.74 $\left.\mu \mathrm{m} / \mu \mathrm{m}^{3}\right)$. The spatial distribution of TPB lines in the sampled volume is shown in Figs.7a-c for the initial microstructure, the evolved microstructure after 200 time steps with a poor wettability, and that with a good wettability, respectively. It is noticeable that the density of TPB lines in Fig. $7 b$ is reduced by comparing Fig.7a, while it is remarkable that the TPB density is significantly increased in Fig.7c. However, a large fraction of the TPB lines shown in Fig.7c are non-percolating (as confirmed in Table 3).

The relationship between the density of percolating TPBs and the performance of the anode is a complicated one and for a real 3-D microstructure requires finite element modelling of the coupled diffusion and electrochemical reaction processes. Nevertheless, it is possible to obtain an estimate of the effect on electrode performance by approximating the electrode as a solid state porous mixed ionic-electronic conducting electro-catalyst. This is a good approximation when the electronic conductivity is much greater than the ionic conductivity, there are no restrictions from gas diffusion and the active depth of the anode is greater than the scale of the microstructure. For a mixed conducting cathode the theory developed by 
Adler, Lane and Steele [S. B. Adler, J. A. Lane, and B. C. H. Steele, "Electrode kinetics of porous mixed-conducting oxygen electrodes," Journal of the Electrochemical Society 143 (11), 3554-3564 (1996).] shows that the electrode polarisation resistance is proportional to $(D k)^{-1 / 2}$, where $D$ is the ionic diffusion coefficient in the solid and $\mathrm{k}$ is the equilibrium rate of exchange per unit surface area of solid for the electrochemical reaction. In the case of a $\mathrm{Ni} / \mathrm{YSZ}$ anode, $D$ refers to ionic diffusion of oxygen in the YSZ and is unchanged in the model of evolution described above. $k$ becomes the equilibrium rate of hydrogen oxidation per unit area of the YSZ surface and is proportional to the density of percolating TPBs, $\rho_{T P B}$. Thus the anode polarisation resistance is proportional to $\left(\rho_{T P B}\right)^{-1 / 2}$. Applying this to the results of the model in Table III indicates that in the poor wettability case the polarisation resistance would be increased by approximately $13 \%$, whereas for the good wettability case the increase would only be approximately $4 \%$.

The modeling results indicate that the good wettability of Ni on YSZ would help in delaying the coarsening and loss of percolating TPB. In other words, good wettability would decrease the degradation rate and enhance the long term electrochemical performance of Ni-YSZ cermet. The wettability of Ni on YSZ is possible to be improved, for example by adding Ti or $\mathrm{TiO}_{2}$ (42). This justifies further fundamental studies on the interfaces and renewed efforts in seeking Ni based alloys with better wettability.

In order to apply the model to predicting the evolution of a real electrode in an operating environment it is necessary to determine the key parameters describing Ni surface energy, $\mathrm{Ni} / Y S Z$ interfacial energy and the relationship between real time increments and the model time steps. These can be determined either by observation of an aged electrode to calibrate the model, or by separate experiments on well-defined model structures (such as patterned surfaces). If, as assumed here, the Ni transport is via the vapour phase, the rate of transport (reflected in the model time step) will be determined by the total equilibrium vapor pressure 
of Ni-containing vapour species. Therefore once the time step is calibrated for one operating condition $\left(\mathrm{P}_{\mathrm{H} 2}, \mathrm{P}_{\mathrm{H} 2 \mathrm{O}}\right.$ and $\left.\mathrm{T}\right)$, thermodynamic modelling can be used to extend this to other conditions. Application of the model to an actual aged anode based on this approach is in progress but is beyond the scope of this paper and will be the subject of a separate paper in the future.

\section{Summary}

A new meso-scale interface interaction model is introduced to calculate the local free energy increase caused by local surface and interface curvature. It is shown that this interaction model is consistent with the classical continuum theory. This CA model, combined with a probabilistic transition rule, has been employed to simulate the well-known phenomena of wetting and grain boundary grooving. It is shown that this new modeling approach can not only reproduce what classical theories predict, but also provide more fundamental understanding of the complex microstructure changes when the systems do not satisfy the highly restrictive assumptions underlying the classical theories.

It has been demonstrated that this approach can be applied to model the complicated topological and morphological changes occurring during ageing of a real microstructure of porous Ni cermet SOFC anode. The modeling results indicate that, at least in the initial stage of ageing, the microstructure changes are very sensitive to the wettability of Ni on YSZ. A better wettability of $\mathrm{Ni}$ on YSZ leads to a slower coarsening of the Ni phase and a slower decrease of the percolating TPB length density. A poor wettability leads to faster coarsening and faster loss of percolation.

In order to use this approach to predict long term microstructural changes in real fuel cell anodes it will be necessary to calibrate the key model parameters (mismatch coefficient and time steps) using additional experimental data from either model experiments or observations on aged anodes. 


\section{Acknowledgements}

The authors would like to thank Dr. Rongshan Qin for the useful discussions and valuable suggestions.

This work was carried out as part of the European Union's Seventh Framework Programme (FP7/20017-1013) for the Fuel Cells and Hydrogen Joint Undertaking (under grant agreement 256885, Solid Oxide Fuel Cells - Integrating Degradation Effects into Lifetime Prediction Models) and the UK Supergen consortium project on "Fuel Cells: Powering a Greener Future" (EPSRC Grant EP/G030995/1).

\section{References}

1. A. Atkinson, S. Barnett, R. J. Gorte, J. Irvine, A. J. McEvoy, M. Mogensen, S. C. Singhal and J. Vohs, Nature materials, 3, 17 (2004).

2. L. Holzer, B. Iwanschitz, T. Hocker, B. Münch, M. Prestat, D. Wiedenmann, U. Vogt, P. Holtappels, J. Sfeir and A. Mai, J. Power Sources, 196, 1279 (2011).

3. P. Tanasini, M. Cannarozzo, P. Costamagna, A. Faes, J. Van Herle, A. Hessler - Wyser and C. Comninellis, Fuel Cells, 9, 740 (2009).

4. Y. Liu and C. Jiao, Solid State lonics, 176, 435 (2005).

5. N. P. Brandon, S. Skinner and B. C. Steele, Annual Review of Materials Research, 33, 183 (2003).

6. J. R. Wilson, W. Kobsiriphat, R. Mendoza, H.-Y. Chen, J. M. Hiller, D. J. Miller, K. Thornton, P. W. Voorhees, S. B. Adler and S. A. Barnett, Nature materials, 5, 541 (2006).

7. P. Shearing, J. Gelb and N. Brandon, Journal of the European Ceramic Society, 30, 1809 (2010).

8. N. Vivet, S. Chupin, E. Estrade, T. Piquero, P. Pommier, D. Rochais and E. Bruneton, Journal of Power Sources, 196, 7541 (2011).

9. P. Costamagna, P. Costa and V. Antonucci, Electrochim. Acta, 43, 375 (1998).

10. A. loselevich, A. A. Kornyshev and W. Lehnert, Solid State lonics, 124, 221 (1999).

11. J. Golbert, C. S. Adjiman and N. P. Brandon, Industrial \& Engineering Chemistry Research, 47, 7693 (2008).

12. M. P. Anderson, G. S. Grest and D. J. Srolovitz, Philosophical Magazine Part B, 59, 293 (1989).

13. D. Raabe, Acta Mater., 48, 1617 (2000).

14. A. D. Rollett, D. J. Srolovitz, R. D. Doherty and M. P. Anderson, Acta Metallurgica, 37, 627 (1989).

15. H. W. Hesselbarth and I. R. Gobel, Acta Mater., 39, 2135 (1991).

16. C. F. Pezzee and D. C. Dunand, Acta Metall. Mater., 42, 1509 (1994).

17. V. Marx, F. R. Reher and G. Gottstein, Acta Mater., 47, 1219 (1999).

18. L. Q. Chen, Annu. Rev. Mater. Res., 32, 113 (2002). 
19. H.-Y. Chen, H.-C. Yu, J. Scott Cronin, J. R. Wilson, S. A. Barnett and K. Thornton, Journal of Power Sources, 196, 1333 (2011).

20. D. Fan, L. Q. Chen and S. P. P. Chen, J. Am. Ceram. Soc., 81, 526 (1998).

21. H. J. Frost and C. V. Thompson, Acta Metallurgica, 35, 529 (1987).

22. Z. Jiao and N. Shikazono, Journal of The Electrochemical Society, 160, F709 (2013).

23. L. Holzer, B. Iwanschitz, T. Hocker, B. Münch, M. Prestat, D. Wiedenmann, U. Vogt, P. Holtappels, J. Sfeir, A. Mai and T. Graule, Journal of Power Sources, 196, 1279 (2011).

24. T. Toffoli, Physica D: Nonlinear Phenomena, 10, 117 (1984).

25. D. Raabe, Annu. Rev. Mater. Res., 32, 53 (2002).

26. J. V. Neumann, Theory of Self-Reproducing Automata, p. 388, University of Illinois Press, London (1966).

27. D. Raabe and L. Hantcherli, Comput. Mater. Sci., 34, 299 (2005).

28. D. Raabe, Acta Mater., 52, 2653 (2004).

29. Y. Liu, T. Baudin and R. Penelle, Journal Name: Scripta Materialia; Journal Volume: 34; Journal Issue: 11; Other Information: PBD: 1 Jun 1996, Medium: X; Size: pp. 1679 (1996).

30. Y. He, H. Ding, L. Liu and K. Shin, Mater. Sci. Eng. A., 429, 236 (2006).

31. H. L. Ding, Y. Z. He, L. F. Liu and W. J. Ding, J. Cryst. Growth, 293, 489 (2006).

32. P. J. P. Pimienta, E. J. Garboczi and W. Craig Carter, Computational Materials Science, 1, 63 (1992).

33. D. P. Bentz, P. J. P. Pimienta, E. J. Garboczi and W. C. Carter, CELLULAR AUTOMATON SIMULATIONS OF SURFACE MASS-TRANSPORT DUE TO CURVATURE GRADIENTS - SIMULATIONS OF SINTERING IN 3-D, p. 413, Materials Research Soc, Pittsburgh (1992).

34. D. Raabe, Philosophical Magazine A, 79, 2339 (1999).

35. D. Raabe and R. C. Becker, Modelling and Simulation in Materials Science and Engineering, 8, 445 (2000).

36. J. W. Bullard, J. Appl. Phys., 81, 159 (1997).

37. R. Tao, M. A. Novotny and K. Kaski, Physical Review A, 38, 1019 (1988).

38. T. Vicsek, Phys. Rev. Lett., 53, 2281 (1984).

39. C. Herring, The Physics of Powder Metallurgy, McGraw-Hill Book Co., New York (1951).

40. J. Laurencin, R. Quey, G. Delette, H. Suhonen, P. Cloetens and P. Bleuet, Journal of Power Sources, 198, 182 (2012).

41. P. R. Shearing, J. Golbert, R. J. Chater and N. P. Brandon, Chem. Eng. Sci., 64, 3928 (2009).

42. A. Tsoga, A. Naoumidis and P. Nikolopoulos, Acta Mater., 44, 3679 (1996). 


\section{TABLES}

TABLE 1. Quantitative 3-D microstructural parameters for Ni-YSZ anodes

\begin{tabular}{lcccccc}
\hline Microstructure & $\mathbf{V}_{\mathbf{N i}}(\boldsymbol{\%})$ & $\mathbf{V}_{\mathbf{Z r}}$ (\%) & $\mathbf{V}_{\text {pore }}(\boldsymbol{\%})$ & $\begin{array}{c}\mathbf{S A}_{\mathbf{N i}}{ }^{*} \\
\left(\mu \mathrm{m}^{2} / \mu \mathrm{m}^{3}\right)\end{array}$ & $\begin{array}{c}\mathbf{S A z r} \\
\left(\mu \mathrm{m}^{2} / \mu \mathrm{m}^{3}\right)\end{array}$ & $\begin{array}{c}\mathbf{A}_{\text {int }} \\
\left(\mu \mathrm{m}^{2} / \mu \mathrm{m}^{3}\right)\end{array}$ \\
\hline Real initial & 35 & 43.4 & 21.6 & 0.65 & 1.16 & 1.43 \\
\hline $\begin{array}{l}\text { Simulated after 200 } \\
\text { time steps }\left(C_{\text {mis }}=0.8\right)\end{array}$ & 35 & 43.4 & 21.6 & 0.54 & 1.42 & 1.17 \\
\hline $\begin{array}{l}\text { Simulated after 200 } \\
\text { time steps }\left(C_{\text {mis }}=0.2\right)\end{array}$ & 35 & 43.4 & 21.6 & 0.53 & 1.02 & 1.58 \\
\hline
\end{tabular}

* area per unit total analysed volume

TABLE 2. Equivalent particle size for different phases in 3-D microstructures

\begin{tabular}{lccc}
\hline Microstructure & Deq $_{\text {of }} \mathbf{N i}(\boldsymbol{\mu} \mathbf{m})$ & Deq of YSZ $(\boldsymbol{\mu m})$ & Deq of Pore $(\boldsymbol{\mu m})$ \\
\hline Real initial & 1.01 & 1.0 & 0.72 \\
\hline $\begin{array}{l}\text { Simulated after 200 time } \\
\text { steps }\left(C_{m i s}=0.8\right)\end{array}$ & 1.23 & 1.0 & 0.66 \\
\hline $\begin{array}{l}\text { Simulated after 200 time } \\
\text { steps }\left(C_{m i s}=0.2\right)\end{array}$ & 0.99 & 1.0 & 0.84 \\
\hline
\end{tabular}

TABLE III.Triple phase boundary densities in 3-D microstructures

\begin{tabular}{lcc}
\hline Microstructure & Percolating TPB density $\left(\boldsymbol{\mu m} / \boldsymbol{\mu m}^{\mathbf{3}}\right)$ & Total TPB density $\left(\boldsymbol{\mu m} / \boldsymbol{\mu \mathbf { m } ^ { 3 } )}\right.$ \\
\hline Real initial & 5.16 & 6.14 \\
\hline $\begin{array}{l}\text { Simulated after } 200 \\
\text { time steps }\left(C_{m i s}=\right.\end{array}$ & 4.06 & 5.09 \\
$0.8)$ & & \\
\hline $\begin{array}{l}\text { Simulated after 200 } \\
\text { time steps }\left(C_{\text {mis }}=\right.\end{array}$ & 4.74 & 10.6 \\
$0.2)$ & & \\
\hline
\end{tabular}




\section{Figure captions}

Fig.1, a) A schematic of a discretized feature in a 2-D microstructure composed of two different phases, red and blue. The cells hatched within the grid are the interface cells for the red phase. The cell ' $\mathrm{X}$ ' is the one in consideration in the text. The mask consists of 4 layers, with the number indicating in which layer the cells are interacting with the target cell. b) A circle representing a 2-D phase with a radius of 70 cells. c) A grey-scale map showing the calculated relative structural imbalance $(\boldsymbol{\Delta u})$ of the cells on the circle surface, note that $\boldsymbol{\Delta u}$ is normalised with the maximum corresponding to 1 and the minimum $\Delta \boldsymbol{u}$ corresponding to 0 in the intensity bar. d) Curvature dependence of the calculated surface energy for different circle radii (values of $n$ ).

Fig.2, a) Initial state of two circular particles of phase $\alpha$ sitting on a flat substrate of phase $\beta$. After simulated long term evolution, the morphology changes to b) if the compatibility parameter $C_{\text {com }}=0.2$, to c) if $C_{c o m}=0.5$; and to d) if $C_{\text {com }}=0.8$. e) The contact angle between two phases as a function of their compatibility parameter. The error bar represents the standard deviation of the simulated contact angle and the continuous line represents Young's equation

Fig.3 a) Initial state of two crystal grains intersecting a surface normally in 2-D. After simulation for 400 time steps this evolves to different groove shapes depending on the lattice mismatch parameter (between the two grains) used for modelling: to b) if $C_{m i s}=0.2$; to c) if $C_{m i s}=0.5$; and to d) if $C_{m i s}=0.8$; e) The modelling results (datum points) compared with the classical theory (continuous line) regarding the dependence of the dihedral angle on the lattice mismatch. 
Fig. 4 a) 3-D representation of 2 cubic nickel particles sitting on a square flat YSZ plate. b) The CA modelled microstructure after 200 time steps assuming $C_{m i s}=0.8$. c) The evolved microstructure after 200 time steps assuming $C_{m i s}=0.2$.

Fig.5 A typical 2-D image of a Ni/YSZ SOFC anode obtained from FIB slice and view.

Fig.6 a) Reconstructed 3-D microstructure of a Ni cermet SOFC anode. b) Ni-only microstructure corresponding to Fig.6a. c) The simulated 3D microstructure after 200 time steps assuming $C_{m i s}=0.8$ d) Ni-only microstructure corresponding to Fig.6c. e) The simulated 3D microstructure after 200 time steps assuming $C_{m i s}=0.2$ f) Ni-only microstructure corresponding to Fig.6e.

Fig.7 a) Total TPB lines corresponding to the microstructure shown by Figs. 6a and $6 \mathrm{~b}$. b) Total TPB lines corresponding to the microstructure given by Figs. 6c and 6d. c) Total TPB lines corresponding to the microstructure given by Figs. 6e and $6 \mathrm{f}$. 
Figures

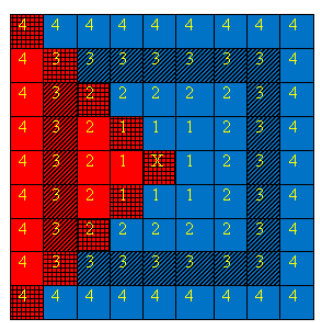

a

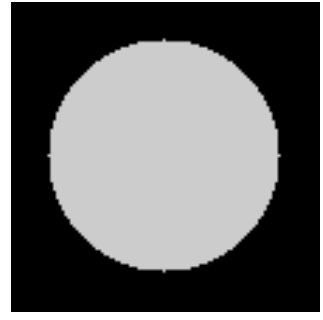

b

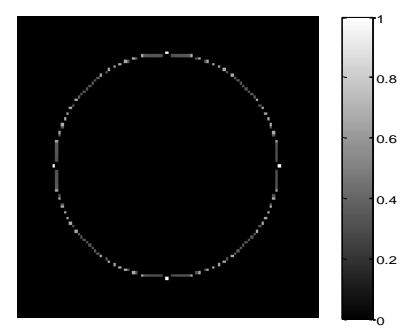

C

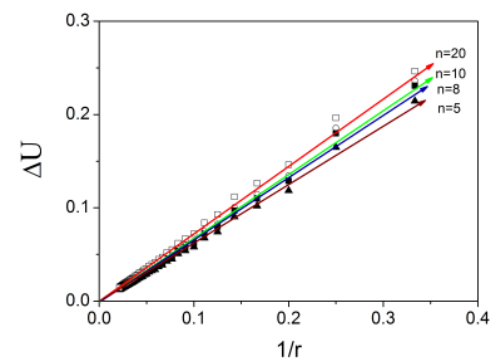

d

Fig.1 


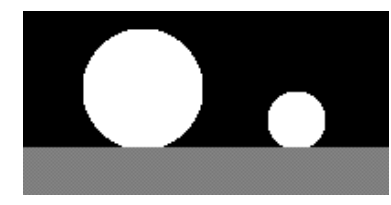

a

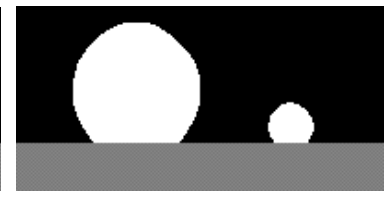

b

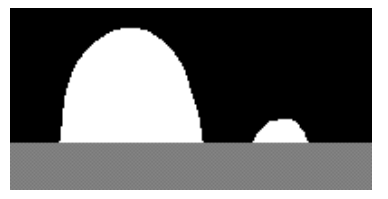

C

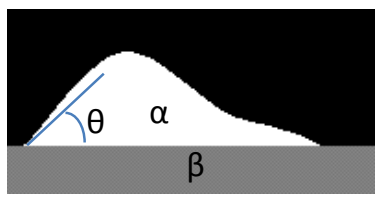

d

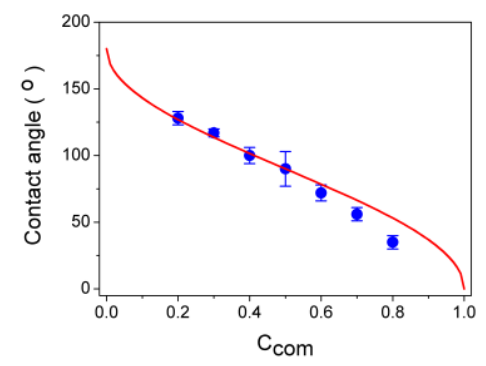

e

Fig.2 


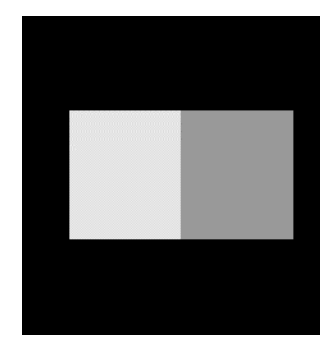

a

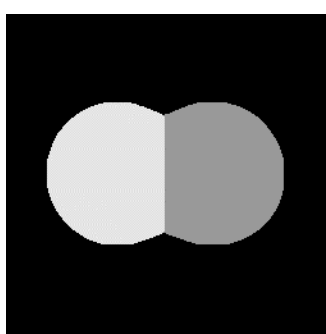

b

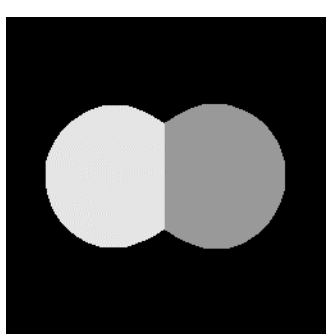

C

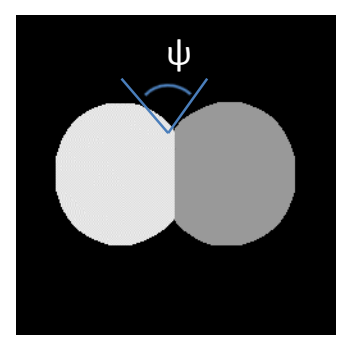

d 


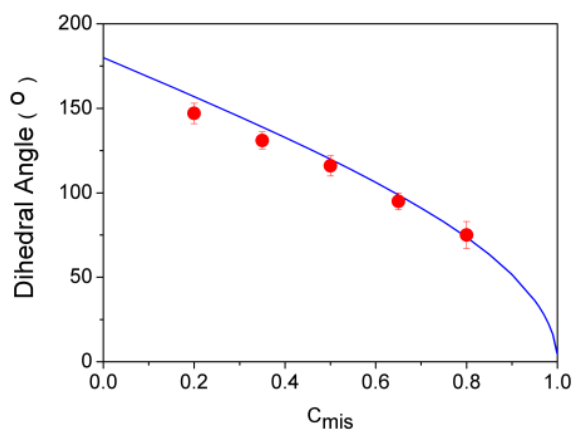

e

Fig.3
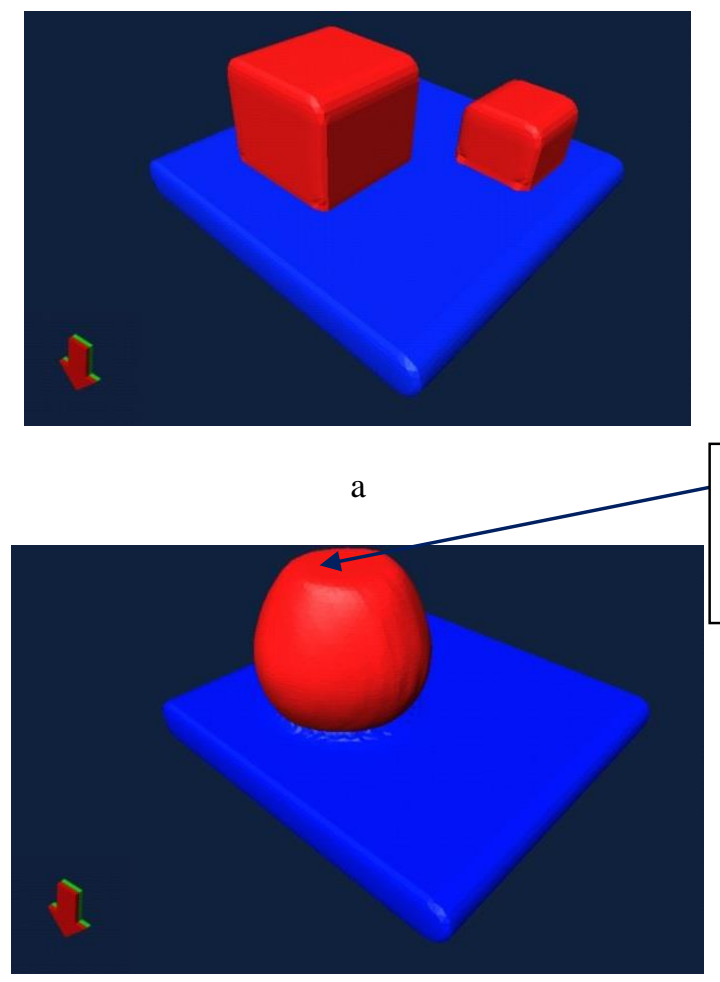

The flat top is due to contact with YSZ above (periodic boundary) 
Fig. 1

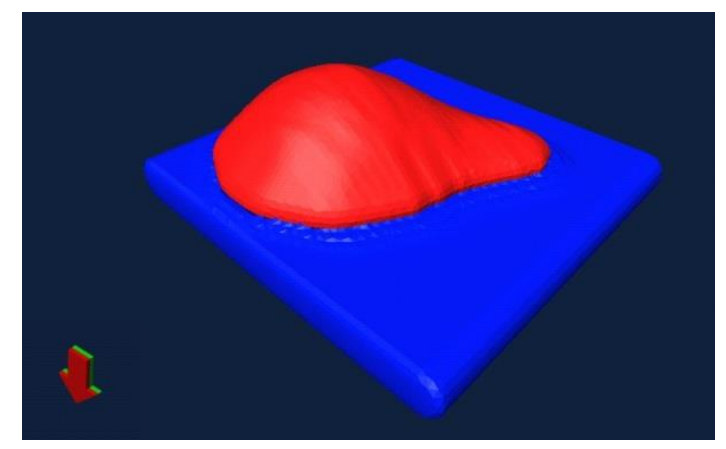

C

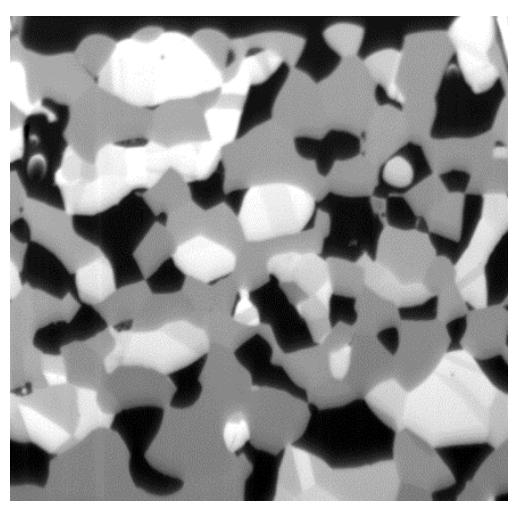

Fig. 5 

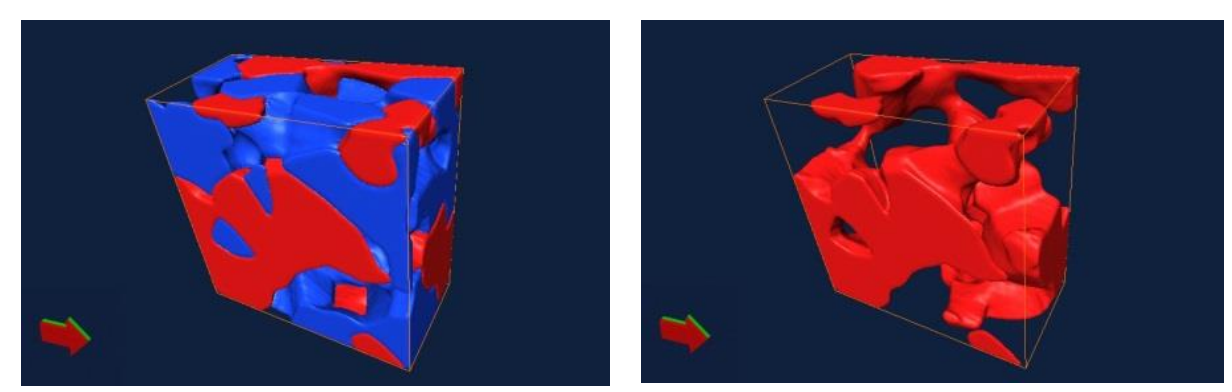

a

b
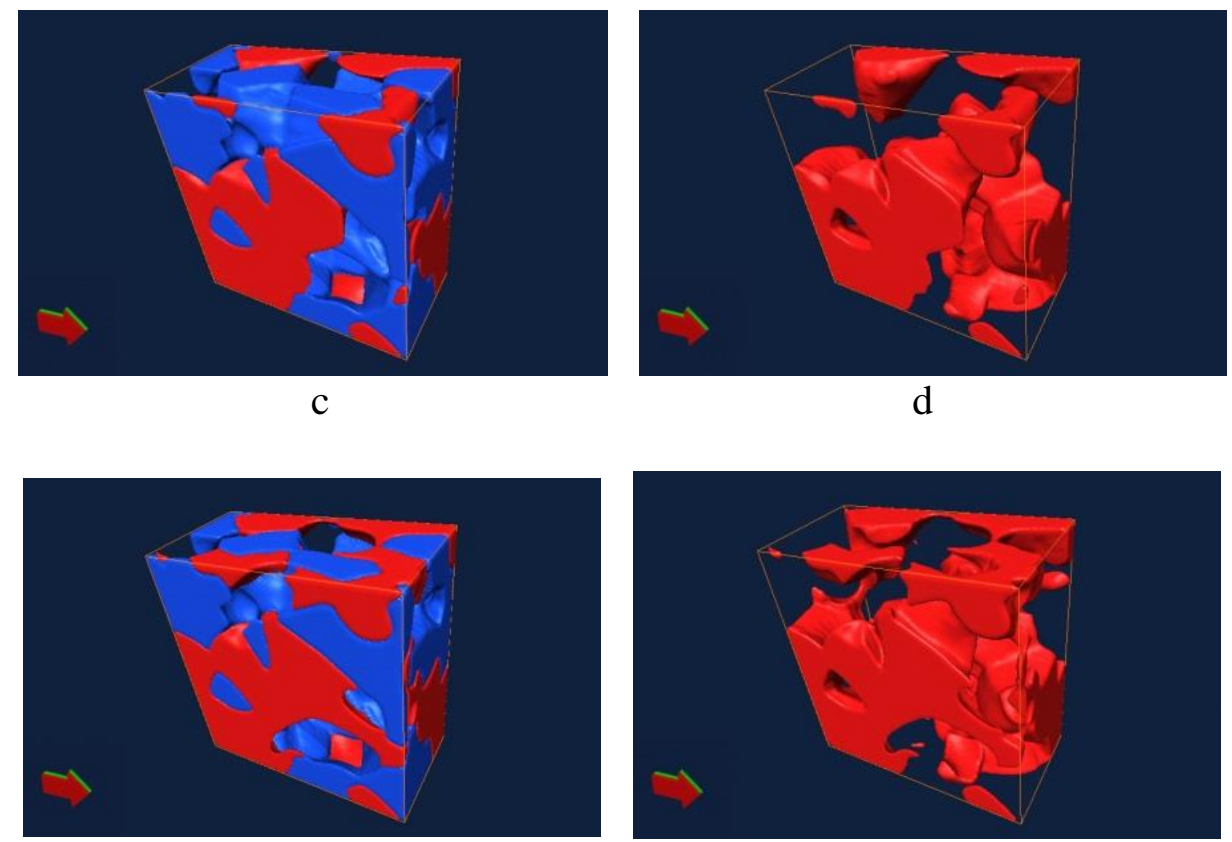

e

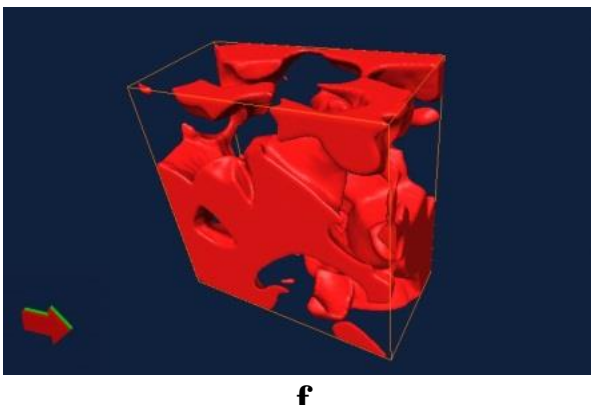

Fig.6 


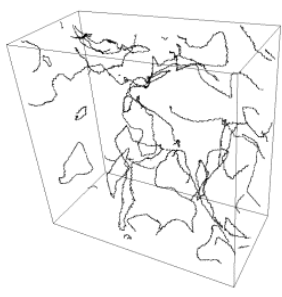

a

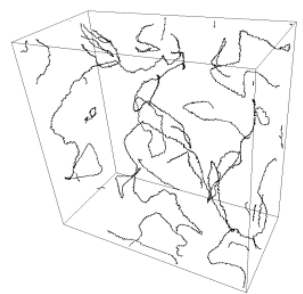

b

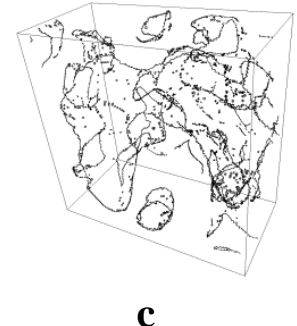

Fig.7 